proposition to most people, who will successfully ignore this moral point and continue to insist on meat as long as it is available. It may not be available indefinitely, however; as the world's population increases, there must come a point where industry will cast an unromantic eye at cow pastures, sheep paddocks and other such inefficient institutions, and will insist on building something useful there.

My own research field is not a biological one, and the following may not be very practical but, I believe, ought to be given some thought: have tissue-culture specialists thought of applying their techniques to the culturing of edible animal tissues? A number of advantages over conventional animal culture spring to mind, the most important one perhaps the fact that, theoretically at least, one should be able to produce more "meat" in a given volume or area than by sending cows out to graze (even if the American cattle industry continues to rationalize its business). The technical problems and costs involved may well be tremendous, but this will become less and less relevant as the population increases. The antikilling moralists would concede, I think, that a mass of cultured animal tissue is (except nutritionally) little different from cultured plant tissue (the hidden moral point-that, given an increasing human population density, we will eventually not be able to allow other animals much space-may be conveniently ignored here).

If the technical problems are soluble, there would be gastronomical advantages in this-there seems to be no reason why one should not culture a vast variety of "meats"; there will be no such thing as a rare and costly tissue-although it is doubtful that this "meat" would turn out like the fibrous stuff we eat now.

I envisage meat factories for the year 2000.

$$
\begin{aligned}
& \text { Yours faithfully, } \\
& \text { D. BRITZ }
\end{aligned}
$$

Jülich,

Germany

\section{Multiple Authorship}

SIR,-In this day of "publish or perish" one increasingly encounters papers authored by several persons. A high percentage of team work, and thus a high percentage of multiple authorship, can, in fact, be considered a reflexion of the state of advancement of a particular science $^{1}$. The January 30,1970 , issue of Science $^{2}$ reporting on the scientific results of the Apollo 11 moon expedition is an excellent though exceptional case in point. In this issue there were 144 papers authored by a total of 619 persons, an average of 4.3 authors per paper. One paper was by 18 authors (is this a record?), two other papers by 14 authors each, one by 12 , and two by 11 each; at the other extreme, ten papers were authored by only one person. Merely the names and addresses on the paper with 18 authors required five column inches of space.

The very number of publications listed in a bibliography of a scientist often gives an inflated estimation of the scientific contribution of that person. Obviously more entries are possible if a person participates in a great deal of team work. What is needed is some method to rate the equivalent value of a scientific paper authored by several persons. Each paper, no matter by how many authors, should count as unity (one equivalent paper). That is, the paper with 18 authors, if listed in bibliographies by each of the 18 authors, should count as one paper total, and not 18. The following table presents sample equivalent values for papers with

\begin{tabular}{|c|c|c|c|c|c|c|}
\hline \multirow{3}{*}{$\begin{array}{l}\text { Paper } \\
\text { authored } \\
\text { by: }\end{array}$} & \multicolumn{6}{|c|}{$\begin{array}{c}\text { Values of equivalent papers } \\
\text { per author }\end{array}$} \\
\hline & & & & hor: & & \\
\hline & A & B & L & & $E$ & $F$ \\
\hline & 1.00 & & & & & \\
\hline & 0.67 & 0.33 & & & & \\
\hline & 0 & 0. & 0.20 & 0.10 & & \\
\hline & 0.33 & 0. & 0.20 & 0 . & 0. & \\
\hline & 0 & & 0.19 & 0.14 & 0.09 & 0.0 \\
\hline
\end{tabular}
up to six authors:

For example, three papers individually authored by " $\mathrm{X}$ " (total of 3 equivalent papers) are "worth" slightly more than six papers authored by "Y" as follows: $Y, Y B, Y B, A Y, A B Y, A B C Y$ (total of 2.94 equivalent papers), even though " $Y$ " has twice as many publications as " $\mathrm{X}$ ".

There are two possibilities for situations with six or more authors per paper since the contribution of the sixth and additional authors ranges from $1 / 21$ to $1 / \infty$ (euphemism for essentially zero: for example, the contribution of author number 18 is $1 / 171$ or 0.006 equivalent paper): (1) the contributors in excess of five might well (preferably!) be relegated to acknowledgment status in a footnote, or (2) they might be listed alphabetically (as is currently done with the more notable movie stars in epics).

A final plea: in personal bibliographies of scientists, entries for papers by several authors should include a list of the authors in the sequence they appear on the paper. Thus, in a bibliography for author "Y" a paper by "ABY" should be cited as "by ABY" and not, as is so commonly done, as "with AB", since the latter gives no indication of the ranking of the authors (and who did all the work).

I leave it to other workers to develop more exact and complex relationships taking into consideration other significant variables (length, type of paper (for example, taxonomic monograph, review paper), etc.).

Yours faithfully,

RudolF ScHMID

Department of Botany,

University of Michigan,
Ann Arbor,

Michigan 48104

Manten, A. A., Earth Sci. Rev., 6, 181 (1970).

2 Science, 167, 417 (1970).

\section{Cycles in Behaviour}

SIR,- The whole approach of analogizing between the natural sciences and the behaviour of human society could well distinguish itself only by its naivety. Nevertheless, history is strewn with examples of the fertility of cross-disciplinary activities, and at least one Great Man has urged us to "only connect".

Young and Ziman (Nature, 229, 91; 1971) concern themselves with establishing a nomenclature to facilitate discussion of cycles in social behaviour, by borrowing terms from physics. This they do very convincingly except that they do not make the important distinction between an oscillating function of time and a periodic function of time. An oscillating function is normally understood to be one which exhibits a sequence of turning points: thus one speaks of super-critically and sub-critically damped harmonic motion, where the former exhibits a monotonic trend toward some asymptote and is non-oscillatory, and the latter is oscillatory but not periodic. A periodic function would exhibit a waveform that is exactly repeated over intervals of the period.

The distinction between oscillations with regularly spaced turning points and periodic motion vanishes when the ordinate is non-numerable in the sense that an event can only be said to occur or not occur, for then only the intervals between events matter. However, there clearly exist cases where more quantifcation of a social variable is possible. For instance, as the authors point out, historical events sometimes display temporal influences that decay in a manner suggestive of a relaxation time. The "modulation" of a periodic function such as the yearly religious festivals by a decaying historical influence could clearly result in an oscillating social variable that is aperiodic.

Perhaps it is sometimes appropriate in discussing the behaviour of human society to use a logarithmic rather than a linear scale of time. The significance to us of a fixed interval of time seems to depend on average roughly how long ago that interval is placed. This follows if events have relaxation times. The "larger" the event and/or the longer its relaxation time the longer its significance: the memory we now have of some interval in history depends on the sum of its remnant influences, and the farther back the interval the less cause, on average, we now have to remember it. History, archaeology, geological eras, scientific papers, personal experience and future forecasting all seem to imply a roughly 